\title{
Oscillation of tissue oxygen index in non-exercising muscle during exercise
}

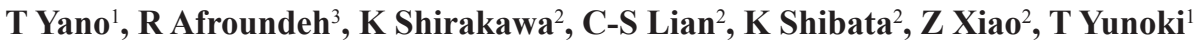 \\ ${ }^{1}$ Department of Human Developmental Sciences, Faculty of Education Hokkaido University, Kita-ku, Japan \\ ${ }^{2}$ Graduate School of Education, Hokkaido University, Kita-ku, Japan \\ ${ }^{3}$ Department of Sport Science and Physical Education, Payame Noor University, Tehran, Iran
}

Received: April 9, 2015

Accepted after revision: June 5, 2015

\begin{abstract}
The purpose of the present study was to examine how oscillation of tissue oxygen index (TOI) in non-exercising exercise is affected during high-intensity and low-intensity exercises. Three exercises were performed with exercise intensities of $30 \%$ and $70 \%$ peak oxygen uptake ( $\dot{\mathrm{V}}_{2}$ peak) for $12 \mathrm{~min}$ and with exercise intensity of $70 \% \dot{\mathrm{V}}_{2}$ peak for $30 \mathrm{~s}$. TOI in non-exercising muscle (biceps brachii) during the exercises for $12 \mathrm{~min}$ was determined by nearinfrared spectroscopy. TOI in the non-exercising muscle during the exercises was analyzed by fast Fourier transform (FFT) to obtain power spectra density (PSD). The frequency at which maximal PSD appeared (Fmax) during the exercise with $70 \% \mathrm{~V}_{2}$ peak for $12 \mathrm{~min}(0.00477 \pm 0.00172 \mathrm{~Hz})$ was significantly lower than that during the exercise with $30 \% \dot{\mathrm{V}}_{2}$ peak for $12 \mathrm{~min}(0.00781 \pm 0.00338 \mathrm{~Hz})$. There were significant differences in blood $\mathrm{pH}$ and blood lactate between the exercise with $70 \% \dot{\mathrm{Vo}}_{2}$ peak and the exercise with $30 \% \dot{\mathrm{V}}_{2}$ peak. It is concluded that TOI in nonexercising muscle oscillates during low-intensity exercise as well as during high-intensity exercise and that the difference in Fmax between the two exercises is associated with the difference in increase in blood lactate derived from the exercise.
\end{abstract}

Keywords: tissue oxygen index, non-exercising muscle, fast Fourier transform, power spectra density, blood lactate

Richard (17) simply explained the mechanism of oscillation in a dissipative structure by a model. The mechanism is represented by chemical equations and can be described as follows:

$$
\begin{aligned}
& \mathrm{G}+\mathrm{A} \rightarrow 2 \mathrm{~A} \\
& \mathrm{~A}+\mathrm{B} \rightarrow 2 \mathrm{~B} \\
& \mathrm{~B} \rightarrow
\end{aligned}
$$

This can be illustrated by translating it to an ecological system where grass $G$ is constantly supplied and animal A eats grass and reproduces as represented by Eq. 1. Animal $\mathrm{B}$ eats animal $\mathrm{A}$ and reproduces in Eq. 2. In Eq. 3 animal B is dying. This system exhibits limit cycle oscillations. The concentrations of A and B oscillate out of phase, i.e., at a high concentration of B, A is low, and a low concentration of A causes B to decrease. Subsequently, the low concentration of B allows A to increase, which is followed by an increase in B, and this continues with a regular pattern. A more detailed introduction to oscillating chemical systems and their mathematical analysis has been represented in previous reports, e.g., in the report by Prigogine (16).

Corresponding author: Yano T

Department of Human Developmental Sciences

Faculty of Education Hokkaido University, Kita-ku, Japan

Phone: 08-11-706-5090; E-mail: yano@edu.hokudai.ac.jp 

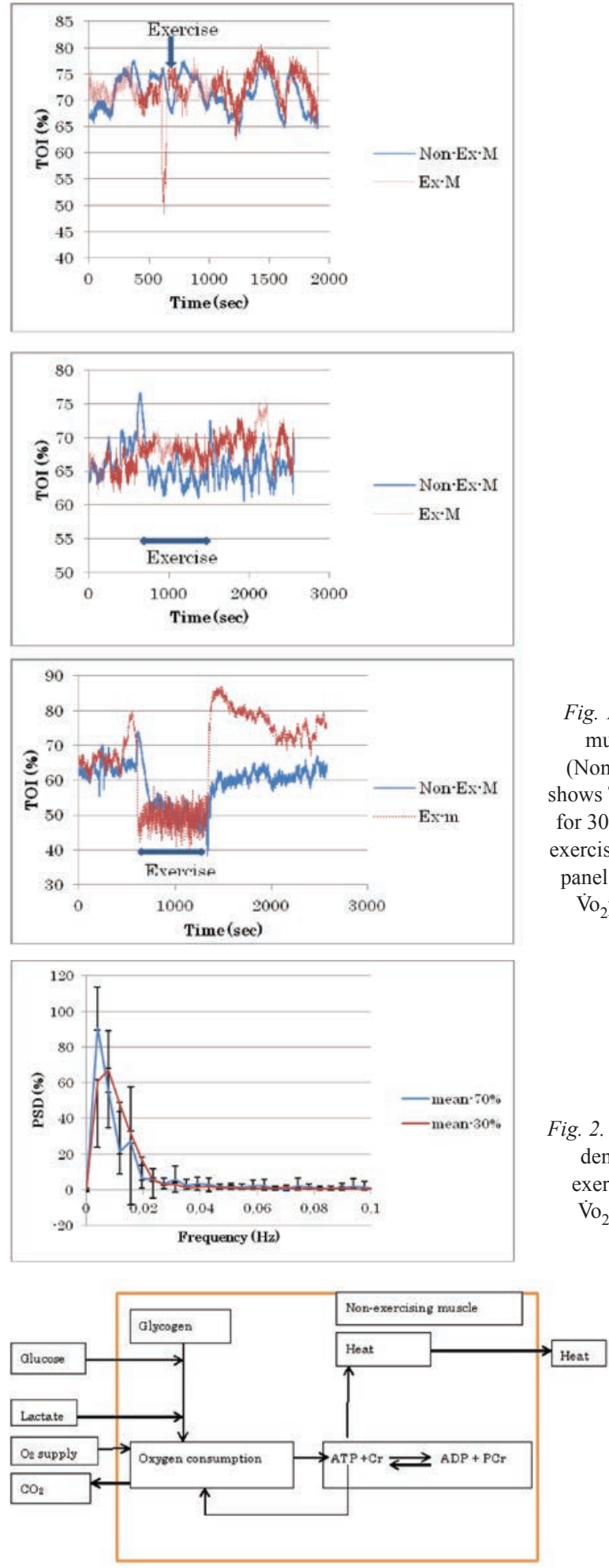

Fig. 1. Tissue oxygen indices (TOIs) in exercising muscle (Ex-M) and in non-exercising muscle (Non-Ex) during three exercises. The upper panel shows TOI kinetics of the exercise with $70 \% \dot{\mathrm{V}}_{2}$ peak for $30 \mathrm{~s}$, the middle panel shows TOI kinetics of the exercise with $30 \% \dot{\mathrm{V}}_{2}$ peak for $12 \mathrm{~min}$, and the lower panel shows TOI kinetics of the exercise with $70 \%$ $\dot{\mathrm{V}}_{2}$ peak for $12 \mathrm{~min}$. Arrows show the period in which exercise was performed

Fig. 2. Mean and standard deviation of power spectra density (PSD) for tissue oxygen index in nonexercising muscle during the exercise with $70 \%$ $\dot{\mathrm{V}}_{2}$ peak for $12 \mathrm{~min}$ and the exercise with $30 \%$ $\dot{\mathrm{V}}_{2}$ peak for $12 \mathrm{~min}$

Fig. 3. A simplified block diagram indicating energetics in the non-exercising skeletal muscle during exercise and input and output of substances in the system. The input of free fatty acid is not shown 
In nonequilibrium thermodynamics, grass $(G)$ is energy supply for the system (input factor). For example, blood glucose and etc. are input factors for skeletal muscle (Fig. 3). These energy sources are consumed to product ATP in oxidative phosphorylation (A). If ATP (B) is a feedback factor on A, A and B could oscillate in the skeletal muscle. The entropy produced in the system is eliminated from the system by death of part of the B (output factor). In the system, free energy is available and consequently the system is self-organized. Such a system affected by input and output is called a dissipative structure.

Sympathetic nervous tone is activated with increasing exercise intensity, and it becomes greater at the exercise-intensities over $60 \%$ of peak oxygen uptake than resting level (18). The enhanced sympathetic nervous activity leads to constriction of arterial vessels, especially these in a non-exercising muscle. In a non-exercising muscle, blood flow is decreased during high-intensity exercise $(2,19)$. As a result, oxygen supply is diminished. However, oxygen consumption is increased (14). In Richard's model, oxygen supply is regarded as an input factor $(\mathrm{G})$ and oxygen consumption is regarded as $\mathrm{A}$. There is an interaction between $\mathrm{A}$ and $\mathrm{B}$, resulting in oscillation of the tissue oxygen index (TOI). A lack of oxygen supply may result in distinct changes in TOI since it has been suggested that an extreme condition induces oscillation of phosphocreatine $(\mathrm{PCr})$ during recovery and that the recovery requires oxygen consumption (7).

The purpose of the present study was to examine how the oscillation of TOI is affected in a non-exercising exercise during high-intensity and low-intensity exercises. The reason why we examined the oscillation of TOI in a non-exercising muscle is as follows. During exercise, consumption of ATP in skeletal muscle is vigorous, and oxidative phosphorylation and glycolysis produce an energy source (ATP). Therefore, the mechanism in the ATP supply system is more complex during exercise than that in a non-exercising muscle. Since simple energetics is easy to analyze, we selected a non-exercising muscle as a research target.

\section{Materials and Methods}

\section{Subjects}

Nine healthy males participated in this study. The means and standard deviations of ages, heights, body weights and peak oxygen uptake ( $\dot{\mathrm{V}}_{2}$ peak) levels of the subjects were $19.6 \pm$ $1.4 \mathrm{yrs}, 169.8 \pm 5.6 \mathrm{~cm}, 63.7 \pm 8.7 \mathrm{~kg}$ and $3.18 \pm 0.52 \mathrm{l} / \mathrm{min}$, respectively. Each subject signed a statement of informed consent following a full explanation regarding the nature of the experiment. The Ethics Committee of Hokkaido University Graduate School of Education approved the present study. This study was performed in accordance with the Declaration of Helsinki.

\section{Experimental protocol}

Each subject performed three leg constant-load exercises and leg incremental exercise until exhaustion on a cycle ergometer (Ergometer 232 CXL, Combi, Tokyo, Japan). After being in a resting state for $4 \mathrm{~min}$, each subject performed constant-load exercise at 20 watts for $4 \mathrm{~min}$, and then work rate was increased by 20 watts each minute until the subject could not maintain the revolution rate of pedaling $(60 \mathrm{rpm})$. $\dot{\mathrm{V}}_{2}$ peak was determined by the maximal value during incremental exercise. In this determination, data of $\dot{V}_{o_{2}}$ for $20 \mathrm{~s}$ were used. On another day, each subject performed three constant-load exercises: exercise with $30 \%$ of $\dot{V}_{2}$ peak determined by incremental exercise for $12 \mathrm{~min}$, exercise with $70 \%$ of $\dot{V}_{2}$ peak for $12 \mathrm{~min}$ and exercise with $70 \%$ of $\dot{\mathrm{V}}_{2}$ peak for $30 \mathrm{~s}$. Each subject rested for $10 \mathrm{~min}$ before the three exercises. The exercises were followed by a recovery period of $20 \mathrm{~min}$. 
Before resting on the cycle ergometer seat prior to three constant-load exercises, each subject sat on a chair to attach electrodes on the subject's chest for monitoring heart rate (HR) and to attach photo probes on the subject's leg and arm (vastus lateralis and biceps brachii) for NIRS. Each subject was instructed to relax and to maintain cycle ergometer cranking in a horizontal position at rest and during recovery on the cycle ergometer. Muscles in the legs are exercising muscles and muscles in the arms are non-exercising muscles.

\section{Measurements and determinations}

Blood samples (each $100 \mu \mathrm{l}$ ) were collected from warmed fingertips using a capillary tube. Each subject's hand was pre-warmed in $40-45^{\circ} \mathrm{C}$ water while sitting on the chair prior to each test in order to arterialize capillary blood (24). After this warming, the subject's hand was warmed by a heating glove at rest, during exercise and during recovery on the cycle ergometer. It has been shown that such blood samples might not accurately reflect arterial $\mathrm{O}_{2}$ pressure but can closely reflect arterial $\mathrm{CO}_{2}$ and $\mathrm{pH}$ (24). Samples were analyzed using a blood gas analyzer (i-STAT1, i-STAT, Abbott Point of Care Inc. IL, USA) to measure $\mathrm{CO}_{2}$ pressure $\left(\mathrm{PaCO}_{2}\right), \mathrm{pH}$ and lactate $(\mathrm{La})$.

Data for respiration gas exchange were obtained using a respiratory gas analyzer by the breath-by-breath mode (AEROMONITOR AE-310S, Minato Medical Science CO., LTD., Osaka, Japan). Ventilation ( $\dot{V E}$ ) was measured by a hot-wire flow meter, and the flow meter was calibrated with a syringe of known volume (2 liters). $\mathrm{O}_{2}$ and $\mathrm{CO}_{2}$ concentrations were measured by a paramagnetic oxygen analyzer and photometric gas analyzer, respectively. The gas analyzer was calibrated by known standard gas $\left(\mathrm{O}_{2}: 15.13 \%, \mathrm{CO}_{2}: 5.068 \%\right)$. Respiration gas exchange was measured continuously during rest, exercise, and recovery periods. Heart rate (HR) was recorded using a heart rate monitor installed in the respiratory gas analyser. $\dot{\mathrm{V}}_{2}$ and $\mathrm{HR}$ were obtained breath-by-breath. In incremental exercise, breath-bybreath data were outputted as 20 s data.

TOIs in the vastus lateralis and biceps brachii were determined using a NIRS system (NIRO200x, Hamamatsu Photonics, K. K. Hamamatsu, Japan). Although NIRO200x can determine both oxygenation by the Modified Beer-Lambert method and TOI determined by the spatially resolved spectroscopy (SRS) method, data obtained by SRS method was used in the present study. The NIRS probe consisted of a light source and an optical detector, with a distance of $3.0 \mathrm{~cm}$ between the light source and detector. Triple-wavelength light $(735,810$ and $850 \mathrm{~nm}$ ) emitted from the light source penetrates tissue, where it is either absorbed or scattered, and some of the scattered light returns to the optical detector. The sampling frequency of TOI was $1 \mathrm{~Hz}$. TOI was calculated from deoxygenation $(\mathrm{HHb})$ and oxygenation $\left(\mathrm{O}_{2} \mathrm{Hb}\right)$ determined by the SRS method using the following equation:

$$
\mathrm{TOI}=\mathrm{O}_{2} \mathrm{Hb} /\left(\mathrm{HHb}+\mathrm{O}_{2} \mathrm{Hb}\right) .
$$

\section{Calculation and statistical analysis}

The data for TOIs during exercise between $5 \mathrm{~min}$ and $10 \mathrm{~min}$ were analyzed by fast Fourier transform (FFT). Power spectral density (PSD) was calculated. PSD at each frequency was individually normalized by the maximal peak value of PSD (PSDmax) by dividing PSD by PSDmax. FFT was not applied for the exercise of $70 \% \dot{\mathrm{V}}_{2}$ peak for $30 \mathrm{~s}$ because the amount of data was insufficient for analysis. 
Results are presented as means \pm standard deviations. Significant differences in levels of blood $\mathrm{pH}$ and blood lactate among the three constant-load exercises were tested by the TukeyHSP method if ANOVA showed significant levels. Blood $\mathrm{pH}$ and blood lactate with time lapse were also examined by Dunnett's test. Frequencies at which PSDmax appeared (Fmax) were compared by the paired $t$-test between TOIs in non-exercising and exercising muscles. The significant level was set at $p<0.05$.

\section{Results}

Tables I and II show changes in blood $\mathrm{pH}$ and lactate in each exercise. In the exercise with $70 \% \dot{\mathrm{V}}_{2}$ peak for $30 \mathrm{~s}$ and the exercise with $30 \% \dot{\mathrm{V}}_{2}$ peak for $12 \mathrm{~min}$, blood $\mathrm{pH}$ and lactate were significantly different from the levels at rest. However, magnitudes of increase in blood lactate and decrease in blood $\mathrm{pH}$ were smaller than those in exercise with $70 \%$ for $12 \mathrm{~min}$. In the exercise with $70 \% \dot{\mathrm{V}}_{2}$ peak for $12 \mathrm{~min}$, blood $\mathrm{pH}$ and lactate were significantly different from the levels at rest. Blood $\mathrm{pH}$ and blood lactate in the exercise with $70 \% \dot{\mathrm{V}}_{2}$ peak were significantly higher than those in other exercises.

Figure 1 shows TOI kinetics in the exercising muscle and non-exercising muscle. In the exercise with $70 \% \dot{\mathrm{V}}_{2}$ peak for $30 \mathrm{~s}$, TOI in the exercising muscle suddenly decreased during exercise and then recovered and oscillated during recovery, and TOI in the non-exercising muscle did not show a sudden decrease, but there was oscillation. In the exercise with $30 \%$ $\dot{\mathrm{V}}_{2}$ peak for $12 \mathrm{~min}$, there was oscillation, but there was a clear decrease during exercise in both exercising and non-exercising muscles. In the exercise with $70 \% \dot{V}_{2}$ peak for $12 \mathrm{~min}$, TOIs in the exercising and non-exercising muscles decreased, but the rate of decrease at the onset of exercise was slower in the non-exercising exercise and TOIs were maintained at low levels during exercise. The low levels were almost the same in exercising and non-exercising muscles. There was oscillation in both muscles.

Figure 2 shows PSD in the non-exercising muscle and exercising muscle. Fmax during the exercise with $70 \% \dot{\mathrm{V}}_{2}$ peak for $12 \mathrm{~min}(0.00477 \pm 0.00172 \mathrm{~Hz})$ was significantly lower than that during the exercise with $30 \% \dot{\mathrm{V}}_{2}$ peak for $12 \min (0.00781 \pm 0.00338 \mathrm{~Hz})$.

Table I. Mean values and standard deviation (SD) of arterialized blood lactate at rest, during exercise (Ex) and during recovery $(\mathrm{Rec})$

\begin{tabular}{|l|l|c|c|c|c|c|c|}
\hline & & Rest & Ex 5 min & Ex 10 min & Rec 5 min & Rec 10 min & Rec 20 min \\
\hline $\mathbf{3 0 \% - 1 2} \mathbf{~ m i n}$ & mean & 1.32 & 1.58 & 1.38 & 1.10 & 1.01 & 1.00 \\
\hline & SD & 0.39 & 0.38 & 0.37 & 0.26 & 0.27 & 0.29 \\
\hline $\mathbf{7 0 \% - 3 0 ~ s e c ~}$ & mean & 1.17 & & & $2.00^{+}$ & $1.65^{+}$ & 1.33 \\
\hline & SD & 0.42 & & & 0.51 & 0.38 & 0.26 \\
\hline $\mathbf{7 0} \%-\mathbf{1 2} \mathbf{~ m i n}$ & mean & 1.01 & $8.10^{*+}$ & $10.21^{*+}$ & $8.97^{*+}$ & $7.40^{* \#+}$ & $4.76^{* \#+}$ \\
\hline & SD & 0.21 & 1.89 & 3.10 & 3.98 & 3.95 & 2.92 \\
\hline
\end{tabular}

* significant difference compared to the exercise with $30 \% \dot{\mathrm{V}}_{2}$ peak for $12 \mathrm{~min}(30 \%-12 \mathrm{~min})$.

\# significant difference compared to the exercise with $70 \% \dot{\mathrm{V}} \mathrm{o}_{2}$ peak for $30 \mathrm{~s}(70 \%-30 \mathrm{sec})$.

${ }^{+}$significant difference compared to the level at rest. $70 \% \dot{\mathrm{V}}_{2}$ peak for $12 \mathrm{~min}$ is expressed as $70 \%-12 \mathrm{~min}$ 
Table II. Mean values and standard deviation (SD) of arterialized blood $\mathrm{pH}$ at rest, during exercise (Ex) and during recovery $(\mathrm{Rec})$

\begin{tabular}{|l|l|c|c|c|c|c|c|}
\hline & & Rest & Ex 5 min & Ex 10 min & Rec 5 min & Rec 10 min & Rec 20 min \\
\hline $\mathbf{3 0 \% - 1 2} \mathbf{m i n}$ & mean & 7.39 & $7.36^{+}$ & $7.37^{+}$ & 7.39 & 7.39 & 7.38 \\
\hline & SD & 0.02 & 0.02 & 0.03 & 0.02 & 0.02 & 0.02 \\
\hline $\mathbf{7 0 \% - 3 0} \mathbf{~ s e c}$ & mean & 7.39 & & & $7.36^{+}$ & 7.38 & 7.38 \\
\hline & SD & 0.01 & & & 0.02 & 0.03 & 0.02 \\
\hline $\mathbf{7 0 \% - 1 2} \mathbf{m i n}$ & mean & 7.39 & $7.27^{* \#+}$ & $7.28^{* \#+}$ & $7.28^{* \#+}$ & $7.30^{* \#+}$ & $7.36^{* \#}$ \\
\hline & & & & & & & \\
\hline & & & & & & & \\
\hline
\end{tabular}

* significant difference compared to the exercise with $30 \% \dot{\mathrm{V}}_{2}$ peak for $12 \mathrm{~min}(30 \%-12 \mathrm{~min})$.

\# significant difference compared to the exercise with $70 \% \dot{\mathrm{Vo}}_{2}$ peak for $30 \mathrm{~s}(70 \%-30 \mathrm{sec})$.

${ }^{+}$significant difference compared to the level at rest. $70 \% \dot{\mathrm{Vo}}_{2}$ peak for $12 \mathrm{~min}$ is expressed as $70 \%-12 \mathrm{~min}$

\section{Discussion}

\section{TOI kinetics in non-exercising muscle}

It is known that sympathetic activity to vessels in the skeletal muscle is increased in highintensity exercise but not in low-intensity exercise $(2,18,19)$. This may induce constriction of vessels not only in non-exercising muscle but also in exercising muscle due to metabolites produced in the exercising muscle. However, in the exercising muscle, constriction of the vessels is inhibited, resulting in an increase in blood flow. In the non-exercising muscle, blood flow is decreased, resulting in a decrease in oxygen supply $(6,15)$. However, it is thought that oxygen supply is not decreased in the exercise with $70 \% \dot{V}_{2}$ peak for $30 \mathrm{~s}$. The level of metabolites in the exercise with $70 \% \dot{\mathrm{V}}_{2}$ peak for $30 \mathrm{~s}$ is not sufficiently high to induce an increase in sympathetic activity due to short duration of exercise. It is also known that oxygen consumption in non-exercising muscle is increased in high-intensity exercise (14). Thus, the decrease in oxygen supply and the increase in oxygen consumption could lead to a decrease in TOI in the non-exercising muscle.

In the exercising muscle, TOI was decreased from the early period of the exercise in high-intensity exercise. This is probably due to the increase in oxygen consumption in the exercising muscle. Indeed, oxygen supply increases from the early period of exercise. However, since the increase in oxygen consumption is greater than that in oxygen supply, TOI is decreased. Thus, the decreases in TOI were almost the same in the non-exercising and exercising muscles, but the oxygen supply level in exercising muscle is thought to be different from that in non-exercising muscle.

\section{Oscillation of TOI in non-exercising muscle}

Eighty percent of lactate becomes an energy source (3). In non-exercising muscle, lactate is taken up (9) as an input factor (Fig. 3). In fact, oxygen consumption is increased in nonexercising muscle during high-intensity exercise (14). In the system of non-exercising muscles, oxidative phosphorylation occurs and ATP could be produced. However, if ATP level is maintained at a constant level (homeostasis), ATP which is increased due to an increase in oxygen consumption in the non-exercising muscle during exercise (14) should be hydrolyzed. This hydrolysis would almost result in production of heat since there is no 
availability of ATP for muscle contraction in the non-exercising muscle. This heat can increase the entropy of the system. This entropy should be eliminated from the system. This system is a dissipative structure.

It has been reported that $\mathrm{PCr}$ recovers from exercise with damped oscillation (7). The frequency of oscillation in that study ranged from $0.002 \mathrm{~Hz}$ to $0.025 \mathrm{~Hz}$ (7). The data obtained in the present study were within this range. PCr re-synthesis during recovery becomes possible by ATP supply by oxidative phosphorylation. ATP is maintained at a constant level even in the active muscle (20). Therefore, homeostasis of ATP is hypothesized (1). In fact, ATP level is maintained constant in exercise (20). For this maintenance, ATP level must be detected by the system. Therefore, we postulate that ATP is a feedback factor for ATP production system. In a non-linear system, due to the time delay of the feedback system, not only ATP but also TOI could be oscillated as mentioned in Introduction. Thus, a dissipative structure is characterized by the oscillation of substances in the system.

\section{Difference in Fmax between the two exercises}

It has been hypothesized that cytosolic $\mathrm{pH}$ affects the frequency of oscillation of $\mathrm{PCr}$ resynthesis during recovery (7). Accordingly, it has been reported that when cytosolic $\mathrm{pH}$ was low, Fmax was low and vice versa. It has been confirmed that Fmax of deoxygenation at rest decreased in the exercising muscle in exercise, (23). However, contradictory results have also been reported (22).

In the present study, this effect on Fmax was confirmed in non-exercising muscle. Sever intramuscular acidosis has been thought to be dominated by lactate from glycolysis. However, it has recently been recognized that in addition to lactate accumulation, changes in the concentrations of strong ion difference (SID), increases in muscular $\mathrm{CO}_{2}$ pressure, and changes in total concentration need to be taken into consideration in the control of $\mathrm{H}^{+}(8)$. In non-exercising muscle during high-intensity exercise, lactate that is increased by highintensity exercise could be taken up by the non-exercising muscle, leading to a fall in the muscle strong ion difference (SID), associated with an increase in muscle $\mathrm{CO}_{2}$ pressure (9). As a result, decrease in cytosolic $\mathrm{pH}$ could occur in non-exercising muscle.

\section{Determination of TOI}

We used TOI determined by spatially resolved spectroscopy (SRS) instead of deoxygenation determined by the Beer-Lambert method (BLM). The estimation of deoxygenation by BLM in previous studies has been reported to be affected by skin blood flow under a certain condition $(4,5)$, though validation of this method has been reported $(12,21)$. Furthermore, skin blood flow is known to oscillate at several bands in frequency $(0.009-1.6 \mathrm{~Hz})$ including a low frequency band $(0.009-0.02 \mathrm{~Hz})(10,11)$. This suggests that skin blood flow affects the frequency of deoxygenation. However, it has been shown that estimation of TOI by SRS is not greatly affected by oxygenation dynamics of skin blood flow (13).

\section{Conclusions}

There were oscillations of TOI in non-exercising muscle in low-intensity exercise as well as in high-intensity exercise. We postulated that ATP was a feedback factor for oxidative phosphorylation and consequently TOI and ATP would oscillate in the non-exercising muscle during exercise. The Fmax of TOI in non-exercising muscle in high-intensity exercise was lower than that in low-intensity exercise, probably being associated with an increase in blood lactate in high-intensity exercise. 


\section{REFERENCES}

1. Balaban RS: Domestication of the cardiac mitochondrion for energy conversion. J. Mol. Cell Cardiol. 46, 832841 (2009)

2. Bevegård BS, Shepherd JT: Reaction in man of resistance and capacity vessels in forearm and hand to leg exercise. J. Appl. Physiol. 21, 123-132 (1966)

3. Brooks GA: Intra- and extra-cellular lactate shuttles. Med. Sci. Sports Exerc, 32, 790-799 (2000)

4. Buono MJ, Miller PW, Hom C, Pozos RS, Kolkhorst FW: Skin blood flow affects in vivo near-infrared spectroscopy measurements in human skeletal muscle. Jpn. J. Physiol, 55, 241-244 (2005)

5. Davis SL, Fadel PJ, Cui J, Thomas GD, Crandall CG: Skin blood flow influences near-infrared spectroscopyderived measurements of tissue oxygenation during heat stress. J. Appl. Physiol. 100, 221-224 (2006)

6. Fadel PJ, Keller DM, Watanabe H, Raven PB, Thomas GD: Noninvasive assessment of sympathetic vasoconstriction in human and rodent skeletal muscle using near-infrared spectroscopy and Doppler ultrasound. J. Appl. Physiol. 96, 1323-1330 (2004)

7. Iotti S, Borsari M, Bendahan D: Oscillations in energy metabolism. Biochimica et Biophysica Acta 1797, 1353$1361(2010)$

8. Jones NL, Heigenhauser GJF: Getting rid of carbon dioxide during exercise. Clin. Sci. (Lond). 90, 323-335 (1996)

9. Kowalchuk JM, Heigenhauser GJF, Lindinger MI, Obminski G, Sutton JR, Jones NL: Role of lungs and inactive muscle in acid-base control after maximal exercise. J. Appl. Physiol. 65, 2090-2096 (1988)

10. Kvernmo HD, Stefanovska A, Bracic M, Kirkebøen KA, Kvernebo K: Spectral analysis of the laser Doppler perfusion signal in human skin before and after exercise. Microvasc. Res. 56, 173-182 (1998)

11. Kvernmo HD, Stefanovska A, Kirkeboen KA, Kvernebo K: Oscillations in the human cutaneous blood perfusion signal modified by endothelium-dependent and endothelium-independent vasodilators. Microvasc. Res. 57, 298-309 (1999)

12. Mancini DM, Bolinger L, Li H, Kendrick K, Chance B, Wilso JR: Validation of near-infrared spectroscopy in humans. J. Appl. Physiol. 77, 2740-2747 (1994)

13. Messere A, Roatta S: Influence of cutaneous and muscular circulation on spatially resolved versus standard Beer-Lambert near-infrared spectroscopy. Physiol. Rep. 1:e00179 (2013)

14. Nagasawa T: Oxygen consumption in nonexercising muscle after exercise. Int. J. Sports Med. 29, 624-629 (2008)

15. Ogata H, Yunoki T, Yano T: Effect of arm cranking on the NIRS-determined blood volume and oxygenation of human inactive and exercising vastus lateralis muscle. Eur. J. Appl. Physiol. 86, 191-195 (2002)

16. Prigogine PE (1979): From Being To Becoming, Time and Complexity in Physical Science. Freeman and Company, San Francisco

17. Richard P: The rhythm of yeast. FEMS Microbiol Rev. 27, 547-557 (2003)

18. Saito M, Kanao Y, Tanaka H, Sakai T: Muscle sympathetic nerve responses during progressive cycling exercise. Adv. Exerc. Sports Physiol. 5, 19-25 (1999)

19. Saito M, Mano T, Iwase S: Changes in muscle sympathetic nerve activity and calf blood flow during static handgrip exercise. Eur. J. Appl. Physiol. Occup. Physiol. 60, 277-281 (1990)

20. Schmitz JPJ, Jeneson JAL, Van Oorschot JWM, Prompers JJ, Nicolay K, Hilbers PAJ, Van Riel NAW: Prediction of muscle energy states at low metabolic rates requires feedback control of mitochondrial respiratory chain activity by inorganic phosphate. PLoS One 7, e34118 (2012)

21. Tran TK, Sailasuta N, Kreutzer U, Hurd R, Chung Y, Mole P, Kuno S, Jue T: Comparative analysis of NMR and NIRS measurements of intracellular $\mathrm{PO}_{2}$ in human skeletal muscle. Am. J. Physiol. 276, R1682-R1690 (1999)

22. Yano T, Afroundeh R, Shirakawa K, Lian C-S, Shibata K, Xiao Z, Yunoki T: Oscillation in tissue oxygen index during recovery from exercise. Physiol. Res. Submitted

23. Yano T, Lian C-S, Arimitsu T, Yamanaka R, Afroundeh R, Shirakawa, Yunoki T: Oscillation of oxygenation in skeletal muscle at rest and in light exercise. Acta Physiol. Hung. 100, 312-320 (2013)

24. Zavorsky GS, Cao J, Mayo NE, Gabbay R, Murias JM: Arterial versus capillary blood gases: a meta-analysis. Respir. Physiol. Neurobiol. 155, 268-279 (2007) 